\title{
Dependability of Fault-Tolerant Systems - Explicit Modeling of the Interactions Between Hardware and Software Components
}

\author{
Karama Kanoun and Marie Borrel* \\ LAAS-CNRS - 7, Avenue du Colonel Roche, 31077 Toulouse Cedex 4 - France \\ E-mail: kanoun@laas.fr
}

\begin{abstract}
This paper addresses the dependability modeling of hardware and software fault-tolerant systems taking into account explicitly the dependency between the various components. These dependencies may result from functional or structural interactions between the components or from interactions due to global system reconfiguration and maintenance strategies. Since the resulting dependencies are stochastic, the dependability of the system cannot be evaluated by combination of the dependability of its components. This paper presents a framework for modeling the dependencies between the various components of a fault-tolerant system. Modeling is based on Generalized Stochastic Petri Nets (GSPNs). The modeling approach is modular: the behavior of each component and each interaction is represented by its own GSPN, while the system model is obtained by composition of these GSPNs. The composition rules are defined and formalized through clear identification of the interfaces between the component and the dependency nets. In addition to modularity, the formalism brings flexibility and re-usability thereby allowing for easy sensitivity studies with respect to the assumptions that could be made about the behavior of the components and the resulting interactions. This approach is applied to a simple, but still representative, example.
\end{abstract}

Key words: Dependability modeling, hardware failures, software failures, interaction between hardware and software components, Petri Nets.

\footnotetext{
* The work presented in this paper has been partially performed while Marie Borrel was with SRTI SYSTEM.
} 


\section{Introduction}

In the context of computer system dependability, the need for addressing simultaneously both hardware and software dependability aspects has now been recognized. However, even though a number of publications have been devoted to the dependability of combined hardware and software systems (see e.g. $[5,6,13,14]$ ), work on both aspects dealt with at the same time is not prevalent. Also, it is noteworthy that, when they are considered together for real-life systems, the interactions between the components are not usually modeled explicitly (see e.g. $[15,16,20])$.

This paper addresses the dependability modeling of hardware and software fault-tolerant systems taking into account the interactions between the various components. These interactions result for example from the communication between the various components for functional purposes (i.e., functional interactions), or from the structure of the system, mainly the distribution of software components onto the hardware components (i.e., structural interactions), or from fault tolerance and maintenance strategies (reconfiguration and maintenance interactions). They induce dependencies between at least two components that are usually stochastic in nature. As a result, system dependability cannot simply be obtained by combining the dependability of its components. An overall model accounting for these dependencies is thus needed. Our aim is to model explicitly these dependencies so as to quantify their influence on system dependability. This is of prime importance during the design of a new system or while upgrading an already existing one. The designer can make different assumptions about the interactions between the components and compare the dependability of the resulting alternative solutions through sensitivity studies. As the nature of interactions is strongly linked to the modeling level considered and the assumptions made at the considered level, it is not possible to model all the interactions that could take place for any fault-tolerant system. Rather, we define a framework for modeling these interactions in a systematic way and, more generally, we define a framework to build up the dependability model of a fault-tolerant system explicitly taking into account these interactions. To do this, we follow a modular approach based on Generalized Stochastic Petri Nets (GSPNs) due to their ability to handle modularity and hierarchy. Note that modular approaches using 
GSPNs or their offsprings are widely used (see e.g., [2, 18] ). Our contribution lies in modeling the interactions between hardware and software components and giving a formal description of these dependencies.

The paper is organized in five sections: Section 2 presents the framework for modeling interactions between hardware and software components. Section 3 gives a formal description of the various types of dependency nets while Section 4 illustrates the approach on a duplex system with several interactions. Section 5 concludes.

\section{Modeling Framework}

The modeling approach consists in identifying, based on the analysis of the system's behavior, dependencies between the components that could be induced by functional or structural interactions or by interactions due to system reconfiguration and maintenance. Some examples of dependencies due to these interactions are given in the following. Error propagation between two software components is an example of stochastic dependency resulting from functional interactions (exchange of data or transfer of intermediate results from one component to another). The halting of the software activities following a permanent failure of the hosting hardware is an example of stochastic dependency induced by a structural interaction. Sharing of a single repairman by the two hardware computers leads to a maintenance dependency whereas switching from an active component (hardware or software) to a spare component following a permanent failure of the active component leads to a reconfiguration dependency. In this paper, we consider interactions that are driven by events occurring in a component whose occurrence may impact the behavior of other components.

A high level model of the system is first derived based on the previous analysis. It is made of blocks and arrows: a block stands for the component model (component net) or a dependency model (dependency net), and an arrow shows the direction of the dependency. The system model is thus obtained by composition of the component and dependency models. In a second step, each block is replaced with its detailed GSPN. To allow for a systematic build up of dependency nets, rules that will have to be followed during model construction are defined. These rules manage the interfaces between 
the dependency and component nets and are prerequisite for modularity, hierarchical modeling and reusability (re-usability is a valuable concept when it comes to doing sensitivity studies about certain assumptions regarding a system's behavior or when several alternative solutions are being considered). Also, these rules allow an easy and fast validation of the global model. In the rest of the section, we give the characteristics of the component and dependency nets and present the various types of dependency nets together with the rules that have to be followed to build up the GSPNs.

\subsection{Component nets}

A component net represents the behavior of a component as resulting from the activation of faults in this component and the subsequent error processing, restart or repair actions. The assumptions made and the degree of detail considered are usually guided by the interactions with other components one wants to exploit (such as the consequences of non detected errors or activation of temporary faults). A component net is designed to verify all the properties of a Petri net: it has its own initial marking, it is live and bounded. It is referred to as a standalone net. It can be connected with dependency nets only following well defined rules as explained hereafter. Connections must not alter the initial basic structure of the component nets: they only consist in adding out going arcs from already existing internal transitions in the component net to places in the dependency net. However the dynamic behavior (transition firing) can be altered by the dependency nets following well specified rules that are presented in the rest of this section.

\subsection{Dependency nets}

A dependency net is linked to at least two adjacent nets: an initializing and a target net that could be component or dependency nets. To formally describe dependency nets and to promote re-usability, we define as much common characteristics as possible. As a result, whatever is the kind of interaction modeled, all the dependency nets are initialized and interfaced with the adjacent nets following the same rules; they only differ in their effects on the target net. The common characteristics and the different effects on the target nets are introduced in figure 1 where a hypothetical dependency net with 
all types of effects identified in our work is given (the notations are introduced with the formalism). These rules are summarized in the following paragraphs.

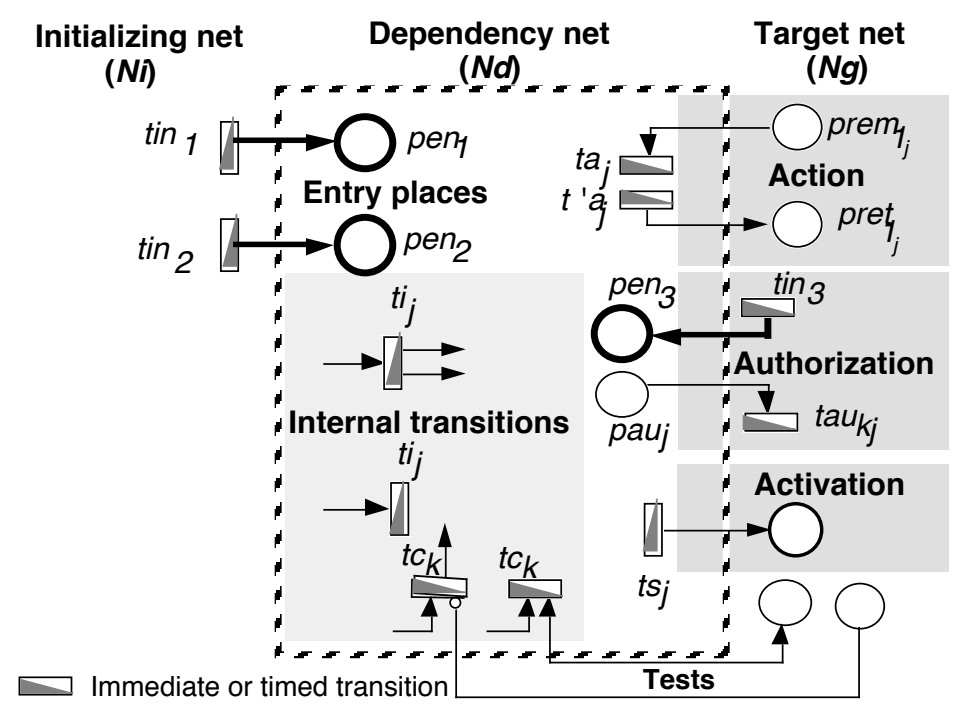

Figure 1: Characteristics of dependency nets

\subsection{Dependency net initialization}

- A dependency net is initialized through the marking of one or more entry places by the initializing net(s), following firing of initializing transitions in these initializing net(s) (as interactions are event driven).

- The initial marking of the entry places is zero.

- When the initializing net is a component net, the consequences of initializing transition(s) firing on the component behavior are modeled within the component net (marking of one or several internal places) and an additional token is generated and deposited in the entry place of the dependency net to activate the interaction.

- If the initializing net is a dependency net, the token deposited in the entry place could be either the one generated when entering the initializing dependency net (this situation corresponds to a series of successive interactions) or an additional one newly generated (corresponding to the initialization in parallel of two or more interactions). 


\subsection{Internal transitions}

The dependency net has internal transitions (timed or instantaneous) whose firing may be independent from the marking of the adjacent nets (independent transitions) or conditioned by the marking of places in the adjacent nets. A condition is modeled by an inhibitor arc or an arc from and towards the tested place: the marking of this place is not changed. An internal transition could be an absorbing transition (i.e., the tokens are removed from the net by the firing of this transition, without putting any token in any place).

\subsection{Interfaces with adjacent nets}

The interfaces with the adjacent nets (excluding initializing arcs and effects on the target nets) are only constituted by tests on the marking of specific places: i. e., they only consist in inhibitor arcs or arcs from and towards the tested place.

\subsection{Effects on target nets}

These effects are strongly related to the type of interaction modeled. Thus, three such effects have been identified:

- If the interaction consists in changing the state of another component (the target net is necessarily a component net), the effect at the GSPN level involves removal of a token from a stable place (i.e., a place followed by timed transitions, this condition stems from the fact that only stable places correspond to states of the components) in the target net and return of the token to the same place or to another stable place of the target net (immediately or after firing internal transitions in the dependency net). This is referred to as an action net.

- If the interaction consists in performing or synchronizing reconfiguration or maintenance actions, the effects depend on the nature of the initializing net:

- if the initializing net is a component net, the interaction consists in coordinating the component restart (or repair) action with the restart (or repair) action of the components to which it is linked: it requests permission before undertaking internal actions, these actions are enabled by 
the dependency net either immediately (firing of immediate transitions in the dependency net) or after a given time (firing of timed transitions in the dependency net). One of the target nets is necessarily the same as the initializing net. At the GSPN level, the effect consists of enabling a transition in the component net through the marking of a place in the dependency net. Since the component net is a standalone net, this means that, in the component net, this transition has also to be enabled by the marking of at least an internal place. This is an authorization net,

- if the initializing net is a dependency net, the interaction consists in activating another interaction with other linked components; at the GSPN level, this consists of initializing another dependency net by depositing a token in its entry place following the firing of an initializing transition in the initializing dependency net. As previously stated, depending on whether the token deposited in the target net is an additional one, both dependency nets run in parallel or in series. This is an activation net.

The previous rules are intended to manage the static links between dependency and adjacent nets. Further rules have to be considered to control the dynamic behavior of the nets (i.e., the tokens generation and their flow). They are given together with the formalism in the next section. Note that model construction is an iterative process with information flow in both directions from/to dependency nets to/from components nets: in the component models, care should be taken to include potential dependencies.

It is worth noting that a dependency net may belong to more than one category at the same time: for example it can be an action net and an authorization net, or an activation net and an action net.

\subsection{Interaction origin and dependency net type}

The interactions have been attributed to three possible origins: functional, structural and those due to system reconfiguration and maintenance. Functional and structural interactions are usually accompanied by a state change, the associated nets are thus at least action nets. Dependencies due to reconfiguration and maintenance may induce a state change, a coordination between several local actions or authorization of local actions: they could thus be modeled by any kind of dependency nets. 


\section{Formal description and validation of the various nets}

The aim of this section is to give a formal description of the various rules introduced in the previous section and to address model validation. We first give the main notations, the other notations being defined in table 1.

\begin{tabular}{|c|c|}
\hline \multicolumn{2}{|c|}{ General Notations } \\
\hline pen $_{k} \in P_{d}$ & Entry place $(\mathrm{EP})$ of $\mathrm{Nd}$. \\
\hline $\operatorname{tin}_{j} \in T_{i}$ & Transition of $\mathrm{Ni}$ that initializes $\mathrm{Nd}$ by marking an EP \\
\hline$M i_{j}$ & Initializing marking of a dependency net \\
\hline$t i_{j} \in T_{d}$ & Internal independent transition in $\mathrm{Nd}$ \\
\hline$t c_{k} \in T_{d}$ & Internal conditioned transition in $\mathrm{Nd}$ \\
\hline \multicolumn{2}{|c|}{ Interfaces for an action net } \\
\hline$t a_{j}, t^{\prime} a_{j} \in T_{d}$ & Removing and returning action transitions in $\mathrm{Nd}$ \\
\hline prem $_{k_{j}} \in T_{g}$ & Input place in $\mathrm{Ng}$ of the removing action transition $t a_{j}$ \\
\hline pret $_{k_{j}} \in T_{g}$ & Output place in $\mathrm{Ng}$ of the returning action transition $t^{\prime} a_{j}$ \\
\hline \multicolumn{2}{|c|}{ Interfaces for an authorization net } \\
\hline$p^{\prime a u_{j}} \in P_{d}$ & Authorization place of $\mathrm{Nd}$ \\
\hline \multicolumn{2}{|c|}{ Interfaces for an activation net } \\
\hline$t s_{k} \in T_{d}$ & Activation transition of $\mathrm{Nd}$ \\
\hline
\end{tabular}

Table 1: Notations

Let $N i, N d$ and $N g$ denote respectively an initializing, a dependency, and a target net, and let $N x$ be any of these nets $(x=i, d, g) . N x=\left(P_{x}, T_{x}, I_{x}, O_{x}, p r_{x}, p a_{x}\right)$ where:

- $P_{x}$ is the set of places of $N x$.

- $T_{x}=\operatorname{Ttim}_{x} \cup \operatorname{Timm}_{x}$ is the set of transitions of Nx:Ttim${ }_{x}$ is the set of timed transitions and Timm $_{x}$ is the set of immediate transitions.

- $I_{x}: P_{x} \times T_{x} \rightarrow \mathrm{N} \cup\{-1\}$ is the input function.

- $O_{x}: T_{x} \times P_{x} \rightarrow \mathrm{N}$ is the output function ( $\mathrm{N}$ is the set of natural integers).

- $p r_{x}$ the set of timed transitions rates, $p a_{x}$ the set of firing probabilities of immediate transitions.

The set of places and transitions are such that:

$P_{i} \cap P_{d}=\varnothing, P_{i} \cap P_{g}=\varnothing, P_{d} \cap P_{g}=\varnothing, T_{i} \cap T_{d}=\varnothing, T_{i} \cap T_{g}=\varnothing$ and $T_{d} \cap T_{g}=\varnothing$ 
The interfaces of a dependency net $N d$ with an initializing net $N i$ and a target net $N g$ are the input and output functions, $I_{i d}, I_{d g}, O_{i d}, O_{d g}$, that connect places and transitions of $N d$ to places and transitions of $\mathrm{Ni}$ or $\mathrm{Ng}$. These functions are defined as follows:

$$
\begin{aligned}
& I_{i d}: P_{i} \times T_{d} \rightarrow \mathbf{N} \cup\{-1\} \\
& I_{d g}: P_{d} \times T_{g} \rightarrow \mathbf{N} \cup\{-1\} O_{i d}: T_{i} \times P_{d} \rightarrow \mathbf{N} \\
& O_{d g}: T_{d} \times P_{g} \rightarrow \mathbf{N}
\end{aligned}
$$

When it is not necessary to distinguish between initializing, dependency and target nets, indices $i, d$, $g$ are omitted.

\subsection{Initialization}

Initializing transitions, entry places and the initializing marking of a dependency net $N d$ are defined as follows: let $\operatorname{tin}_{j} \in T_{i}$ and $p_{k} \in P_{d}$ such that $O_{i d}\left(\operatorname{tin}_{j}, p_{k}\right)>0, I_{d i}\left(p_{k}, t i n_{j}\right)=0$, if $\forall p_{l} \in P_{d}, p_{l} \neq p_{k}$, we have $I_{d i}\left(p_{l}, t i n_{j}\right)=0$ then $\operatorname{tin}_{j}$ is an initializing transition of $N d$ and $p_{k}$ is an entry place of $N d$, denoted $p_{e n}$. An entry place can be initialized by several transitions. In order for a transition $\operatorname{tin}_{j}$ of a net $\mathrm{Ni}$ to be fired, one must have: $\forall p_{m} \in P_{i} \quad I_{i d}\left(p_{m}, t i n_{j}\right) \geq 0 \Rightarrow M i_{n}\left(p_{m}\right) \geq I_{i d}\left(p_{m}, t i n_{j}\right)$ ( $M i_{n}$ is the initializing marking of $N d$ ) and $I_{i d}\left(p_{m}, t i n_{j}\right)=-1 \Rightarrow M i_{n}\left(p_{m}\right)=0^{1}$.

\subsection{Internal transitions}

Internal transitions of a dependency net can be independent or conditioned by the marking of places in adjacent nets. They are defined as follows:

- $t i_{j} \in T_{d}$ is an independent transition if $\forall p_{k}$ such that $I\left(p_{k}, t i_{j}\right)>0$ or $O\left(t i_{j}, p_{k}\right)>0$ then $p_{k} \in P_{d}$, $I=I_{d}, O=O_{d}$.

- $t c_{k} \in T_{d}$ is a conditioned transition if the two following conditions are verified:

1) $\exists p_{j} \in\left(P_{g} \cup P_{i}\right)$ such that: $I\left(p_{j}, t c_{k}\right)=O\left(t c_{k}, p_{j}\right)>0$ or $I\left(p_{j}, t c_{k}\right)=-1$

$1 \quad I(p, t) \geq 0 \Rightarrow M n_{x}(p) \geq I(p, t)$ means that there must be enough tokens in all the input places of $\mathrm{t}$ to enable it. $I(p, t)=-1 \Rightarrow M n_{x}(p)=0$ means that if there is an inhibitor arc from $p$ to $t$, there must be no token in $\mathrm{p}$ to enable $\mathrm{t}$. 
2) $\forall p_{n} \in\left(P_{g} \cup P_{i}\right)$ such that $I\left(p_{n}, t c_{k}\right)>0$ or $O\left(t c_{k}, p_{n}\right)>0$ then $I\left(p_{n}, t c_{k}\right)=O\left(t c_{k}, p_{n}\right)$.

An internal transition can be an absorbing transition. An independent transition $t i_{j}$ or a conditioned transition $t c_{j}$ is an absorbing transition if: $\forall p_{n} \in\{P i \cup P d \cup P g\}, O\left(t i_{j}, p_{n}\right)=0$ or $O\left(t c_{j}, p_{n}\right)=0$.

\subsection{Action nets}

An action net ends with a transition which causes a token to be removed from a stable place of the target net and to be returned to the same or another stable place of the same net. Removal and return can be done through two distinct transitions (with internal transitions and places between them) or through the same transition. The number of tokens in the target net remains unchanged. Transitions $t a_{j}$ and $t^{\prime} a_{j}$ are action transitions if the four following conditions are met:

1) $\forall p_{h} \in P_{i}, I\left(p_{h}, t a_{j}\right)=0$ and $\forall p_{l} \in P_{i}, O\left(t a_{j}, p_{l}\right)=0$

2) $\exists p_{k} \in P_{g}$ such that: $O_{d g}\left(t a_{j}, p_{k}\right)=0$ and $I_{d g}\left(p_{k}, t a_{j}\right)>0\left(t a_{j}:\right.$ removing transition $)$ or: $\exists p^{\prime}{ }_{k} \in P_{g}$ such that: $O_{d g}\left(t^{\prime} a_{j}, p^{\prime}{ }_{k}\right)>0$ and $I_{d g}\left(p^{\prime}{ }_{k}, t^{\prime} a_{j}\right)=0\left(t^{\prime} a_{j}\right.$ : returning transition $)$

3) $\sum_{i=1}^{\left|P_{g}\right|} O\left(t^{\prime} a_{j}, p_{i}^{\prime}\right)=\sum_{i=1}^{\left|P_{g}\right|} I\left(p_{i}, t a_{j}\right)$

4) $p_{k}$ is a stable place: $\exists t \in$ Ttimg $_{g}$ such that $I_{d}\left(p_{k}, t\right)>0$ and $\forall t \in$ Timm $_{g}$ such that $I_{d}\left(p_{k}, t\right)>0$ then $I_{d}\left(p_{k}, t\right)=O_{d}\left(t, p_{k}\right), p^{\prime}{ }_{k}$ must verify an equivalent relation.

$p_{k}$ is then the input place of the removing transition $t a_{j}$, being denoted $\operatorname{prem}_{k j}$ and $p^{\prime}{ }_{k}$, the output place of the returning transition $t^{\prime} a_{j}$, is denoted $\operatorname{pret}_{k j}$.

\subsection{Authorization nets}

An authorization net ends with one or several places enabling firing of transitions in the target net(s), authorization places. In this case, the target net is necessarily the initializing net.

$\operatorname{pau}_{j} \in P_{d}$ is an authorization place of $N d$ if:

$\forall t \in T_{d}$ such that $I\left(\right.$ pau $\left._{j}, t\right)=0$ then $\exists t_{j_{k}} \in\left(T_{i} \cup T_{g}\right)$ such that $I\left(\right.$ pau $\left._{j}, t_{j_{k}}\right)>0$ and $O\left(t_{j_{k}}\right.$, pau $\left._{j}\right)=0 ; t_{j_{k}}$ is then the authorized transition in $N c$, it is denoted $t a u_{k j}$. 


\subsection{Activation nets}

An activation net allows linking of dependency nets (i.e. synchronize the related interactions). It ends with a transition, synchronization transition, that sends one or several tokens in one or several other dependency nets (but does not remove tokens from these nets). $t s_{j} \in T_{d}$ is an activation transition if $\forall p_{m} \in P_{g} I_{g d}\left(p_{m}, t s_{j}\right)=0$ and if $\exists p_{k} \in P_{g}$ such that $O_{d g}\left(t s_{j}, p_{k}\right)>0$.

\subsection{Dynamic behavior}

The generation, the flow and absorption of the tokens has to be controlled while building up a dependency net so as to ensure that the resulting global net is bounded and live. Each token, generated upon dependency net initialization by the marking of an entry place must thus be removed either in the dependency net itself or through its effect on the target net (i. e., when leaving the net). It is then necessary that as long as a dependency net place is marked, whatever the global marking, there is a transition that can be fired and that removes a token from this place. This condition must be formalized for the internal places of all types of dependency nets.

Let $P_{y}$ be such that $P_{y}=P_{d}$ for action and activation nets, $P_{y}=\left\{P_{d}-P_{a u t}\right\}$ for authorization nets. The internal places and transitions of a dependency net must satisfy:

1)Every place has at least one transition that removes tokens: $\forall p \in P_{y} \exists t \in T_{d} \mid I(p, t)>0$ and $O(t, p)=0$

2)If there exists an arc with multiplicity $x$ from a place to a transition, there exist $x-1$ other arcs with multiplicity 1 to $\mathrm{x}-1$, from the same place to $\mathrm{x}-1$ other transitions with the same input and output as the preceding transition:

$\forall t \in T_{d}$, and $p \in P_{y}$ with $I(p, t)>0$ and $O(t, p)=0$ if $\exists p^{\prime} \in P_{d}$ such that $I\left(p^{\prime}, t\right)=x, x \in \mathbf{N}$ then $\exists x$ transitions $t_{j} \in T_{d}$ such that $I\left(p^{\prime}, t_{j}\right)=j, j=1, \ldots, x, I\left(p, t_{j}\right)>0$ and $O\left(t_{j}, p\right)=0$.

All these transitions are independent internal transitions.

3) if there exists a test arc with multiplicity $\mathrm{x}$ between a place and a transition, there exist $\mathrm{x}-1$ other arcs with multiplicity 1 to $\mathrm{x}-1$, from the same place to $\mathrm{x}-1$ other transitions with the same input and output as the preceding transition. 
$\forall t \in T_{d}$, and $\forall p \in P_{y}$ with $I(p, t)>0$ and $O(t, p)=0$, if $\exists p^{\prime} \in P \quad$ such that $I\left(p^{\prime}, t\right)=O\left(t, p^{\prime}\right)=x$, then $\exists x$ transitions $t_{j}$ such that $I\left(p^{\prime}, t_{i}\right)=O\left(t_{j}, p^{\prime}\right)=j, j=1, \ldots, x \quad I\left(p, t_{j}\right)>0$ and $O\left(t_{j}, p\right)=0$.

If $p^{\prime} \in P_{d}, t$ is an independent transition, if $p^{\prime} \notin P_{d}, t$ is a conditioned transition.

4) If there exists an inhibitor arc from a place to a transition then there must exist an arc from the same place to another transition.

$\forall t \in T_{d}$ and $p \in P_{y}$ with $I(p, t)>0$ and $O(t, p)=0$, if $\exists p^{\prime} \in P$ such that $I(p, t)=-1$, then $\exists t^{\prime}$ such that $I\left(p^{\prime}, t^{\prime}\right)=0, I\left(p, t^{\prime}\right)>0$ and $O\left(t^{\prime}, p\right)=0$.

if $p^{\prime} \in P_{d}, t$ is an independent transition, if $p^{\prime} \notin P_{d}, t$ is a conditioned transition.

5) The sum of firing probabilities of immediate transitions in conflict is always equal to 1:

$\forall p \in P_{d}$, if there are $\left\{t_{1} \ldots t_{i} \ldots t_{u}\right\} \subset$ Timm $_{d}$ such that $I\left(p, t_{i}\right)>0$, and $\forall p^{\prime}$ such that $I\left(p^{\prime}, t_{i=x}\right)=$ constant

and $I\left(p^{\prime}, t_{i \neq x}\right)=0$, then $\sum_{\mathrm{i}=1}^{\mathrm{n}} \mathrm{pa}\left(\mathrm{t}_{\mathrm{i}}\right)=1$. If $p^{\prime} \in P_{d}, t$ is an independent transition, if $p^{\prime} \notin P_{d}, t$ is a conditioned transition.

6)In some situations, depending on the marking of the target net, the token must be removed by an absorbing transition if it cannot be removed by the target or initializing net: $t \in T_{d}$ is an absorbing transition if $\forall p \in\{P i \cup P d \cup P g\}, O(t, p)=0$.

\subsection{Model validation}

Several verifications are needed to reach confidence in the model; they are usually grouped into two categories: syntactic and semantic validation [10]. Syntactic validation consists in checking that the model represents the dependability of a system; it mainly includes structural validations. Semantic validation consists of checking that the model represents the dependability of the system under validation; it requires comparing the system and the model behaviors with respect to variations of the underlying assumptions. Usually it is performed through sensitivity studies. Due to the scope of the paper, we concentrate on the structural validation. The rules for interfacing dependency and component nets and for managing the dynamic behavior allow us to obtain, by construction models that are structurally valid (i.e., live and bound). Structural validation is progressively done, starting from the 
component nets and gradually adding dependency nets. Identification of possible problems is thus easy. Also, these verifications can be achieved automatically by computation of place- and transitioninvariants for checking necessary or sufficient conditions of liveliness and boundedness with a tool such as SURF-2 [3] developed at LAAS-CNRS.

\section{Application to the duplex system}

Let us consider a duplex system composed of two hardware computers (H1 and H2) and two identical software replicas: each replica is implemented on a computer. We assume semi-active replication [17]: the leader replica (L) processes all input messages and provides output messages while the follower replica (F) does not produce output messages. The internal state of $\mathrm{F}$ is updated by means of notifications from L completed by direct processing. Temporary faults in the software are tolerated by exception handling mechanisms associated with each replica, whereas the activation of permanent faults leads to restart the replica. To reduce system unavailability, after detection of an error due to a permanent fault in $\mathrm{L}$, the software replicas switch their roles: processing is performed on the new leader before restarting the new follower. If $\mathrm{L}$ and $\mathrm{F}$ fail, $\mathrm{L}$ is restarted first. Also, in case of failure of the hardware hosting L (identified as H1), the replicas switch their roles; the computer hosting the new follower is then repaired. With respect to hardware repair policy when the two computers are in failure, we consider two assumptions:

- R1: the two computers share a single repairman and priority of is given to H1,

- R2: two repairmen are available.

\subsection{High level modeling}

Interactions are directly related to the assumptions made about the components' behavior. Owing to the importance of the impact of temporary faults on the behavior of hardware and software components $[7,8,12,19]$, both permanent and temporary faults are considered in this example.

It is assumed that the activation of a fault may lead to the following dependencies:

- Following activation of a hardware fault: 
- an error due to the activation of a temporary fault in a hardware computer may propagate to the hosted software replica,

- an error due to the activation of a permanent fault in a hardware computer leads to stopping the hosted software replica that is restarted after the end of hardware repair.

- Following activation of a software fault: owing to the notifications sent from the leader to the follower, a non detected error in the leader or an error due to a permanent fault in the leader usually referred to as solid fault - may propagate to the follower (it is assumed that errors due to temporary faults - usually referred to as soft faults — are confined and do not propagate).

Dependencies induced by fault tolerance and maintenance strategies are as follows:

- Between software replicas: dependency due to fault tolerance of permanent software faults, i.e., reconfiguration from $\mathrm{F}$ to $\mathrm{L}$.

- Between hardware computers: dependency due to reconfiguration and repair.

- Between all components: coordination of fault tolerance and maintenance actions to form a global recovery strategy when several components are in failure.

These dependencies are summarized in table 2 together with the names of the associated nets which are used to build up the high level model of the duplex system. The latter is given in figure 2 where $\mathrm{N}_{\text {Hard }}$ and $\mathrm{N}_{\text {Soft }}$ represent a computer and a software replica model respectively.

The corresponding GSPNs are built up following the rules and formal description presented in Sections 2 and 3; they are successively given in the remainder of the section.

\subsection{Hardware and software component nets}

Figure 3 gives the component nets. The hardware model is based on the following assumptions:

- Faults are activated with rate $\lambda_{\mathrm{h}}$.

- With probability $\mathrm{p}_{\mathrm{h}}$ the fault is permanent, (probability of a temporary fault $\left(1-\mathrm{p}_{\mathrm{h}}\right)$ ).

- The effects of an error due to a temporary fault are eliminated within a short time $1 / \delta_{\mathrm{h}}$. 


\begin{tabular}{|l|l|}
\hline$N_{\text {Prop }}$ & $\begin{array}{l}\text { models propagation of a hardware error to the } \\
\text { hosted software replica }\end{array}$ \\
\hline$N_{\text {Stop }}$ & $\begin{array}{l}\text { models software stop after activation of a } \\
\text { permanent fault in the hosting hardware }\end{array}$ \\
\hline$N_{\text {Prop }}^{\prime}$ & models propagation of a software error \\
\hline$N_{\text {Rep }}$ & models hardware reconfiguration and repair \\
\hline$N_{\text {Rec }}$ & models software reconfiguration \\
\hline$N_{\text {Strat }}$ & models the global recovery strategy \\
\hline
\end{tabular}

Table 2: Dependency nets

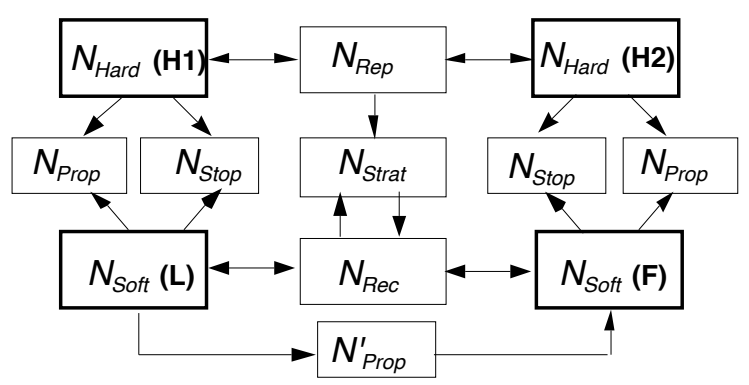

Figure 2: High level model of the duplex system

- An error due to a permanent fault is either detected with probability dh, or non detected (1-dh); error processing rate: th.

- The effects of a permanent, non detected error may be perceived later (perception rate zh).

- The repair rate including software restart (following detection or perception of an error) is $\mathrm{m}$.

Equivalent assumptions are made regarding the behavior of the software replicas:

- Faults are activated with rate $\lambda_{\mathrm{s}}$.

- An error is either detected with probability $\mathrm{d}_{\mathrm{s}}$, or non detected $\left(1-\mathrm{d}_{\mathrm{s}}\right)$; detection rate $\tau_{\mathrm{s}}$.

- The detected error is processed by means of exception handling mechanisms during a short time $1 / \pi_{\mathrm{s}}$. At the end of error processing, 1 ) if the fault is temporary (probability $\left(1-\mathrm{p}_{\mathrm{s}}\right)$ ) its effects are eliminated and the software resumes its normal mode of operation, 2) if the fault is permanent (probability $\mathrm{p}_{\mathrm{s}}$ ); the software has to be restarted (rate: $v$ ) to eliminate its effects.(1- $\mathrm{p}_{\mathrm{s}}$ ) measures the efficiency of fault containment procedures $[8,11]$. 
- The effects of a non detected error may be eliminated (elimination rate $\delta_{\mathrm{S}}$ ), or perceived (perception rate $\zeta_{\mathrm{s}}$ ) in which case the software replica has to be restarted.

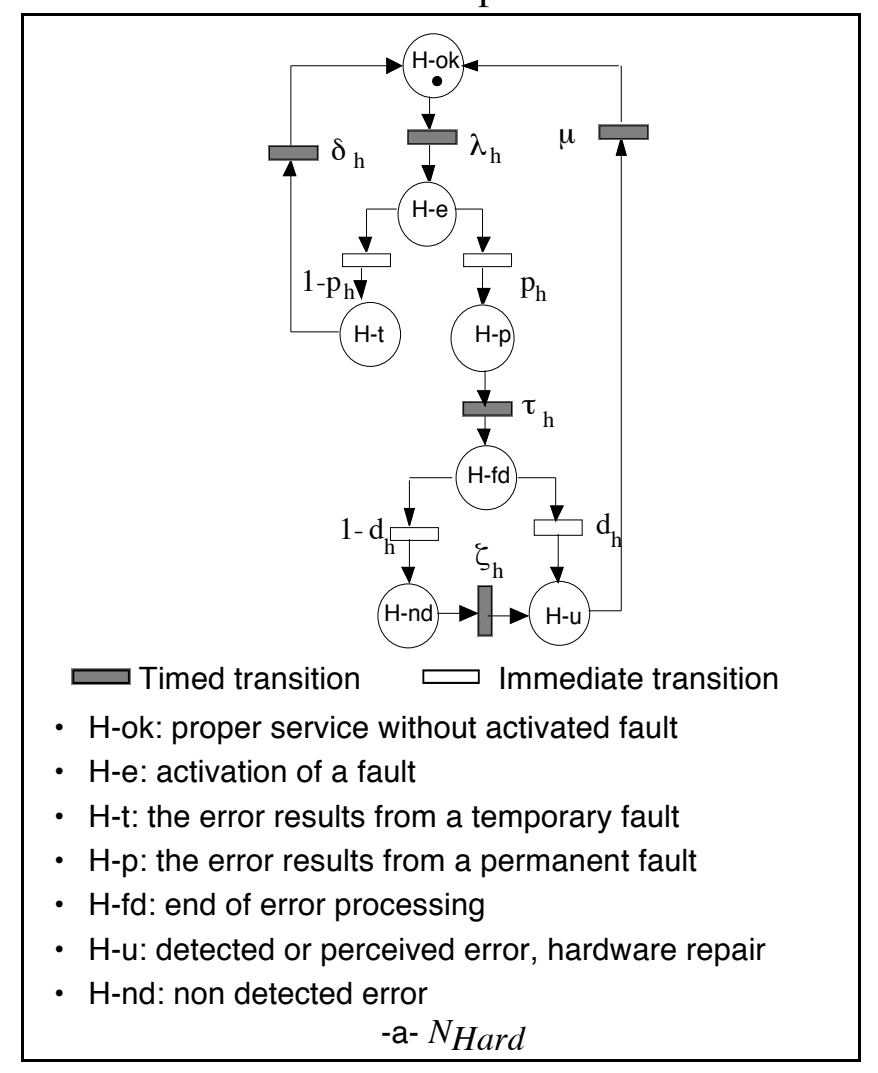




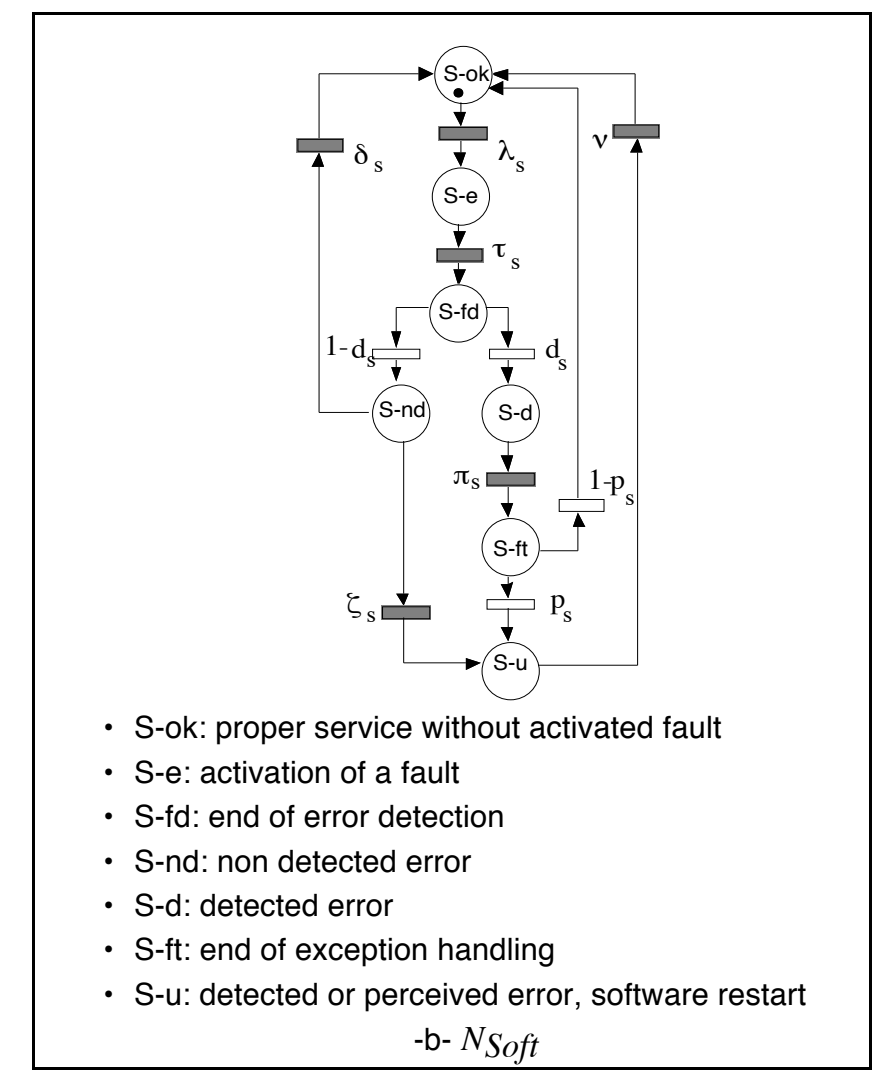

Figure 3: Hardware computer and software replica nets

The difference between these nets lies in that for hardware, temporary and permanent faults are differentiated by their respective consequences following activation, whereas for software, they can only be distinguished after specific processing [12].

\subsection{Error propagation nets}

\section{From hardware to software}

It is assumed that only undetected errors and those due to temporary faults can propagate from a hardware computer to the hosted software replica. The error propagation net, shown in figure 4-a, is initialized by the marking of place Prop following the firing of transition $1-\mathrm{d}_{\mathrm{h}}$ (undetected error) or of transition 1- $\mathrm{p}_{\mathrm{h}}$ (an error due to a temporary fault) in the hardware net (initializing net). With probability $1-\mathrm{p}_{\mathrm{ph}}$, the error is not propagated and with probability $\mathrm{p}_{\mathrm{ph}}$ it is. $N_{\text {Prop }}$ is an action net, whose effects on the software net (target net) are as follows: 
- If the token is in S-ok, it is returned to S-e, the induced error is then processed in the same way as when the fault is activated without propagation (through $\lambda_{\mathrm{s}}$ in figure $3-\mathrm{b}$ ).

- If the token is in S-e, since a fault is already activated in the software, the probability of error detection may be reduced $\left(d_{S}^{\prime} \leq d_{s}\right)$, if the errors are detected, the token is returned to $S-d$; if they are non detected (with probability $1-\mathrm{d}_{\mathrm{S}}$ ) the token is returned to S-nd.

- If the token is in S-nd (an internal error is non detected in the replica) the propagated error and the internal error are detected with probability $\mathrm{d}_{\mathrm{s}}\left(\mathrm{d}_{\mathrm{s}} \leq \mathrm{d}_{\mathrm{s}}\right.$, owing to the perturbation due to the first error) the token is returned to S-d; the errors remain undetected with $1-\mathrm{d}{ }_{\mathrm{s}}$.

- If the token is in S-d the propagated error can compromise error processing and prevent the recovery of an error due to a temporary fault. The internal and propagated errors are recovered with probability $1-p_{p}\left(1-p_{p}<1-p_{s}\right)$.

- If the token is in S-u, the software replica is already under restart, the token of NProp is absorbed through tp-u and the token of $\mathrm{N}_{\text {Soft }}$ is kept in S-u.

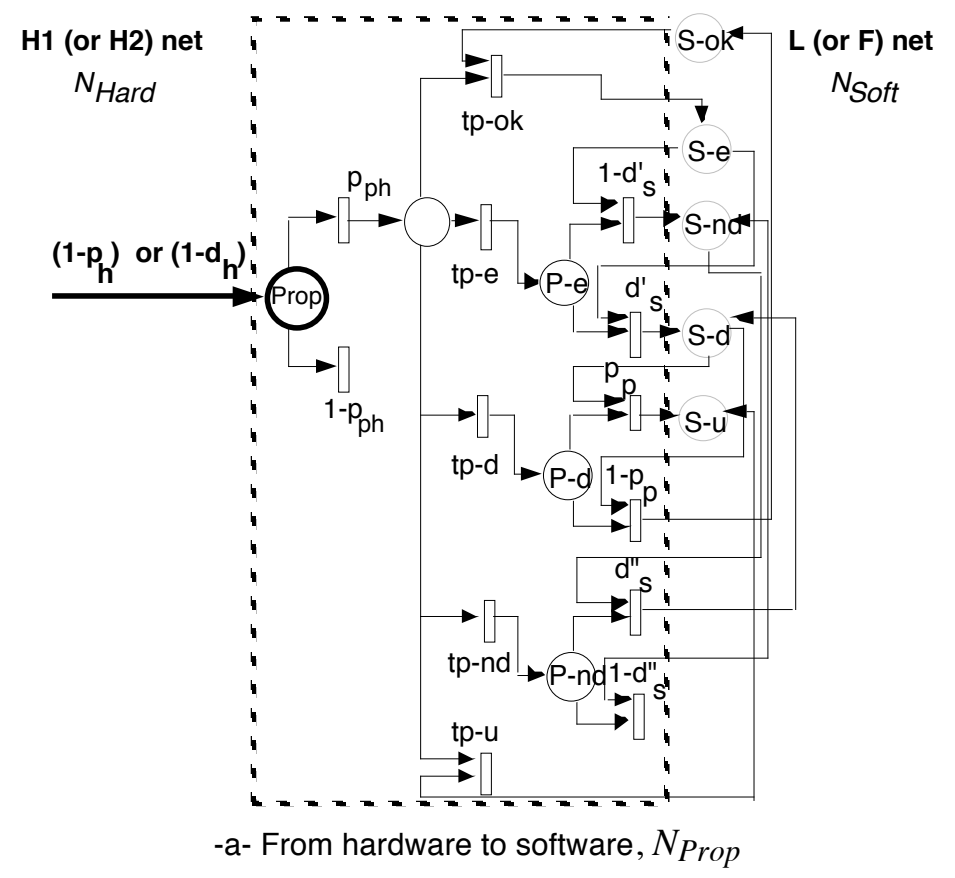




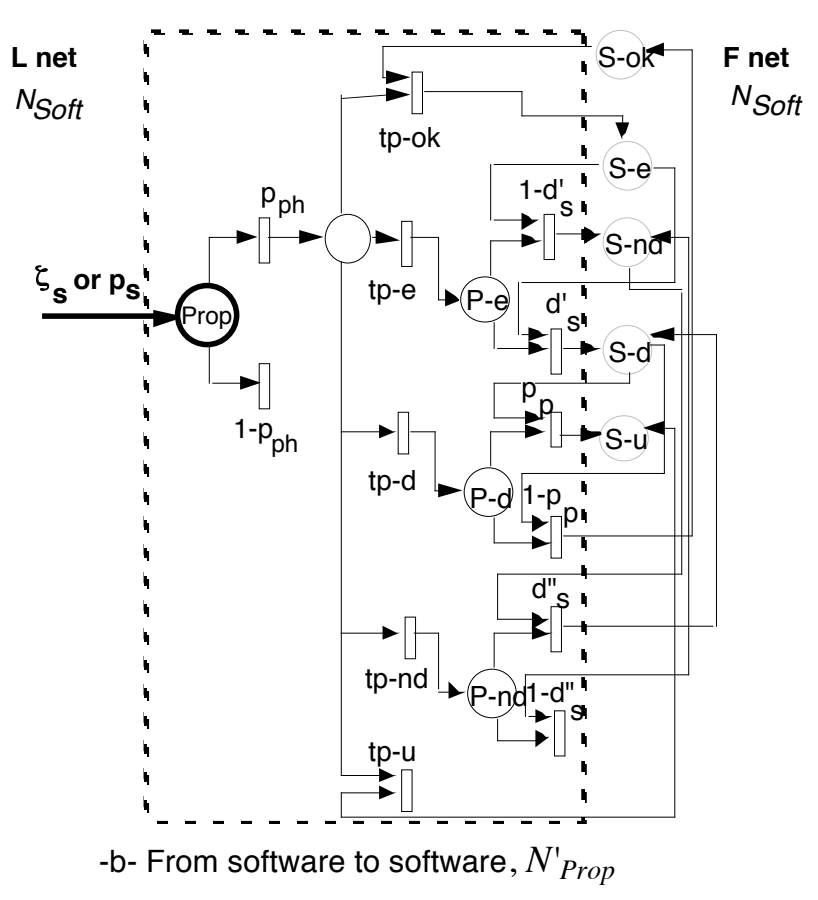

Entry places are indicated in bold; dashed places are defined in another net whose name is indicated in italic.

Initializing arc from an initializing net whose name is given in italic

Figure 4: Error propagation nets

\section{From the software leader to the follower}

The dependency net, the target net and the effects on the target net are exactly the same as for the hardware error propagation net. They differ by the initializing net and the initializing transitions. It is given in figure 4-b. It is assumed that only errors due to the activation of permanent faults in the leader (detected or undetected) can propagate. The error propagation net is thus initialized following the firing of $\zeta_{\mathrm{s}}$ or $\mathrm{p}_{\mathrm{s}}$ in the net of the leader. The probability of error propagation is $\mathrm{p}_{\mathrm{ps}}$.

It is worth noting that at a higher modeling level, error propagation from $\mathrm{L}$ to $\mathrm{F}$ can be regarded as common mode failures: an error in the software leader causes also an error in the follower with a probability $\mathrm{p}_{\mathrm{ps}}$.

\subsection{Software stop and restart net}

Following a detected error or the perception of an undetected error in a hardware computer, the hosted software replica is stopped and is restarted after repair of the hardware. We assume that the repair includes the replica restart. The software stop and restart net (Figure 5) is an action net, it is 
initialized by the marking of STP following the firing of transition $\zeta_{h}$ (perception of a non detected error due to a permanent fault) or $d_{h}$ (detection of an error due to a permanent fault). Transitions t 1 to t5 remove the token from places $\mathrm{S}$-ok, S-e, S-d, S-nd or S-u respectively. After repair of the hardware (including software restart), RST is marked and the token is returned to S-ok (authorization for restart).

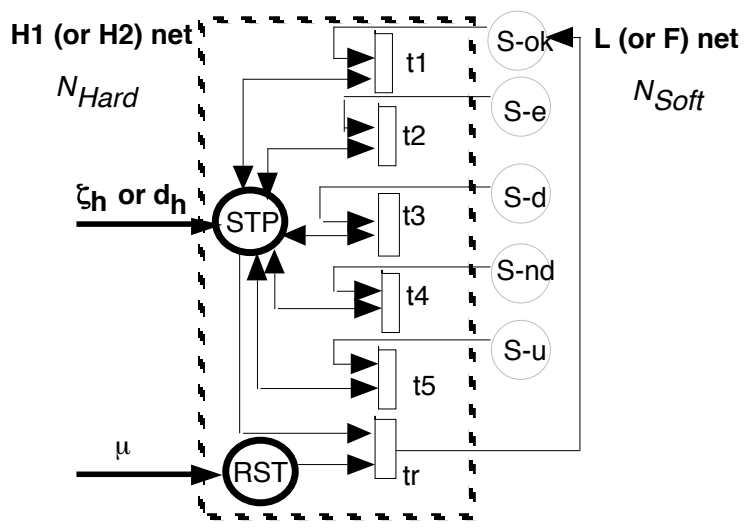

Figure 5: Software stop and restart, $N_{\text {Stop }}$

\subsection{Hardware reconfiguration and repair net}

As previously stated, we consider two different assumptions: R1 assumes a single repairman, while R2 assumes the presence of two repairmen. The corresponding nets are given in figure 6. Each net is composed of two parts corresponding respectively to reconfiguration (the shaded parts on the figures) and repair. They are grouped together because the reconfiguration is automatically followed by a repair. Since the reconfiguration strategy is the same, the associated nets are the same. The two nets are commented together and, when they are different, the repair policy is specified. 

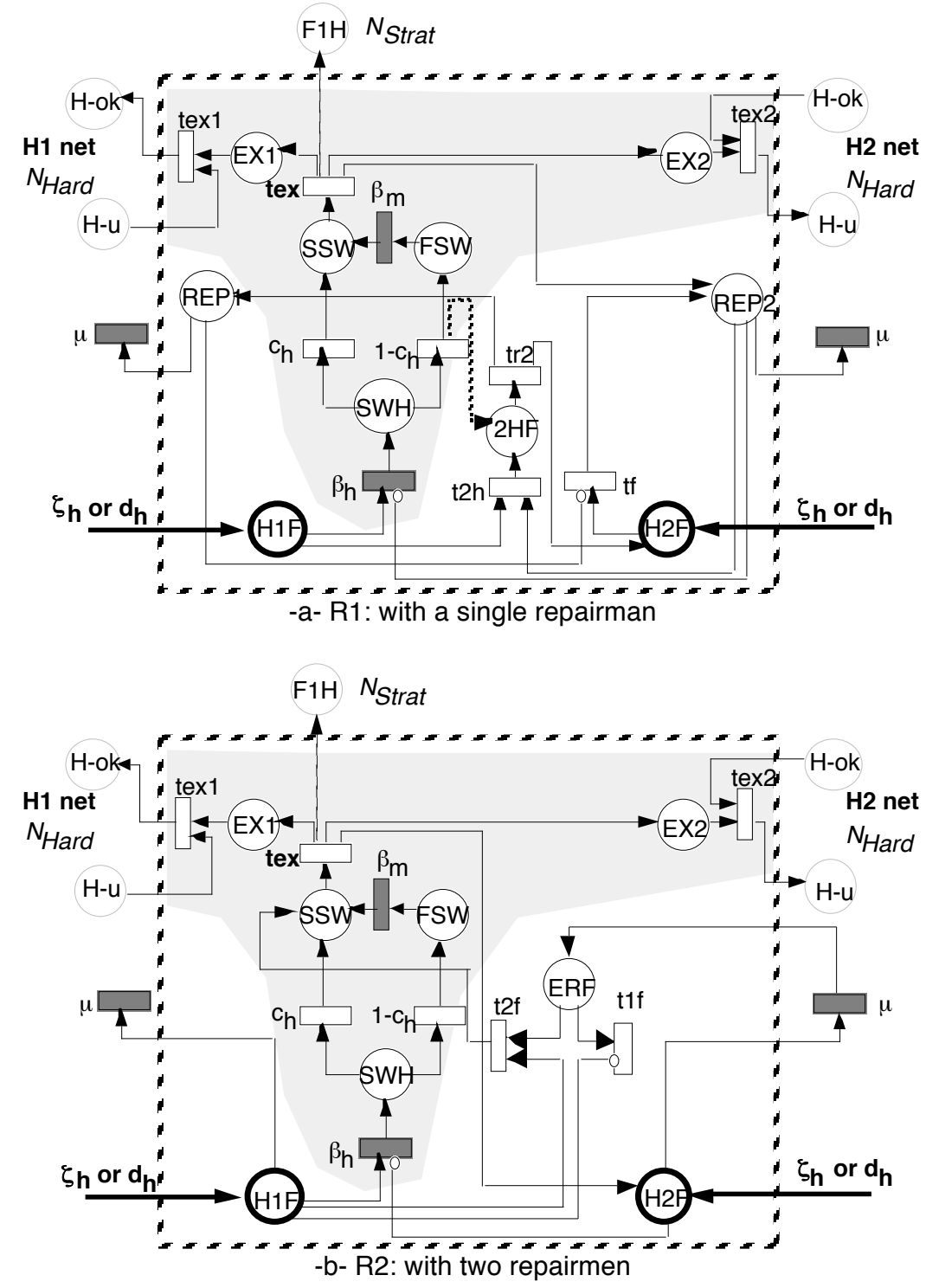

$--\rightarrow$ initializing arc, towards an entry place of a target net whose name is given in italic

Figure 6: Hardware reconfiguration and repair nets, $N_{R e p}$

$\mathrm{N}_{\text {Rep }}$ is initialized by the marking of $\mathrm{H} 1 \mathrm{~F}$ (respectively $\mathrm{H} 2 \mathrm{~F}$ ) following the firing of $\mathrm{d}_{\mathrm{h}}$, detection of an error due to a permanent fault or $\zeta_{\mathrm{h}}$ perception of an undetected error, in the hardware hosting L, H1 (respectively $\mathrm{H} 2)$ :

- if $\mathrm{H} 1 \mathrm{~F}$ is marked, $\mathrm{H} 1$ is in failure (H1 is the initializing net, $\mathrm{H} 2$ the target):

- if H2 is not in failure (REP2 not marked) switching is attempted, $\left(\beta_{\mathrm{h}}\right)$ and HSW is marked:

1) Switch can succeed with probability $c_{h}$, place SSW is then marked, 
2) It can fail with probability $1-\mathrm{c}_{\mathrm{h}}$, FSW is marked and switching is done manually ${ }^{2}\left(\beta_{\mathrm{m}}\right)$, SSW is then marked; tex can be fired, places EX1, EX2 and REP2 are marked; tex1 and tex 2 can be fired, they remove the token from $\mathrm{H}-\mathrm{u}$ to $\mathrm{H}-\mathrm{ok}$, and from $\mathrm{H}-\mathrm{ok}$ to $\mathrm{H}-\mathrm{u}, \mathrm{F}$ becomes the new leader, $\mathrm{L}$ the new follower and it can be restarted (REP2 is marked in figure 6-a for R1, H2F is marked in figure 6-b for R2),

- for $\mathrm{R} 1$ : if $\mathrm{H} 2$ is in failure and under repair (REP2 marked): $\mathrm{t} 2 \mathrm{~h}$ is fired removing the token from REP2 to 2HF; tr2 can then be fired returning a token in REP1 and one in $\mathrm{H} 2 \mathrm{~F}$ in order to repair $\mathrm{H} 1$, then $\mathrm{H} 2$,

- for R2: if $\mathrm{H} 2$ is in failure (H2F marked): repair of $\mathrm{H} 1$ and $\mathrm{H} 2$ are enabled; at the end of $\mathrm{H} 2$ repair, if $\mathrm{H} 1$ is still under repair, the leader is restarted on $\mathrm{H} 2$ (marking of SSW via t2f),

- for $\mathrm{R} 1$ if $\mathrm{H} 2 \mathrm{~F}$ is marked: $\mathrm{H} 2$ is in failure ( $\mathrm{H} 2$ is initializing net and target): if $\mathrm{H} 1$ is not in failure (REP1 not marked), tf can be fired and REP2 is marked, authorizing the repair of H2; else the token stays in $\mathrm{H} 2 \mathrm{~F}$ until the end of $\mathrm{H} 1$ repair; repair of $\mathrm{H} 2$ in then allowed through the marking of REP2 (for R2: repair of $\mathrm{H} 2$ is enabled without any condition on $\mathrm{H} 1$ ).

For R1 and R2: if $N_{R e p}$ is initialed by $\mathrm{H} 1$ only, its is an action, activation and an authorization net; when initialized by $\mathrm{H} 2$ only, it is an authorization net. If it is initialed by $\mathrm{H} 1$ then $\mathrm{H} 2$ (or $\mathrm{H} 2$ then $\mathrm{H} 1$ ) it is an authorization net.

\subsection{Software reconfiguration net}

The software reconfiguration net is given in figure 7 . It is initialized by the marking of S1F (respectively $\mathrm{S} 2 \mathrm{~F}$ ) following the firing of transition $\mathrm{p}_{\mathrm{s}}$, a detected error due to a permanent fault or perception of an undetected error in L (respectively F) through transition $\zeta_{s}$ :

- if $\mathrm{S} 1 \mathrm{~F}$ is marked, $\mathrm{L}$ is in failure ( $\mathrm{L}$ is the initializing net and $\mathrm{F}$ the target):

- if F is not in failure (RSTF not marked) switching is attempted $\left(\beta_{\mathrm{S}}\right)$ and SWS is marked.

2 Other possible assumption: it can be assumed that the manual switch is not attempted. In this case, transition 1-- $c_{h}$ leads to place $2 \mathrm{HF}$ (dashed arc in figure 6-a); place FSW and transition $\beta_{\mathrm{m}}$ have to be omitted. 
1) Switch can succeed with probability $c_{S}$, places EXL, EXF and RSTF are then marked. Marking of EXL allows firing of tex that removes the token from S-u to S-ok in L. Marking of EXF allows the firing of one of transitions t1 to t4 that removes the token in the leader net from places S-ok, S-e, S-d or S-nd and return it to S-u. Marking of RSTF enables transition $\mathrm{v}($ in $\mathrm{F}$ ) to restart it.

2) Switch can fail with probability $1-c_{s}$, places EXF and 2 SF are then marked. Marking of 2SF allows transition tr2 firing that marks places RSTL and S2F. Marking of place RSTL enables transition $v$ in the leader to restart it. Marking of S2F allows the follower restart only after the end of the leader restart.

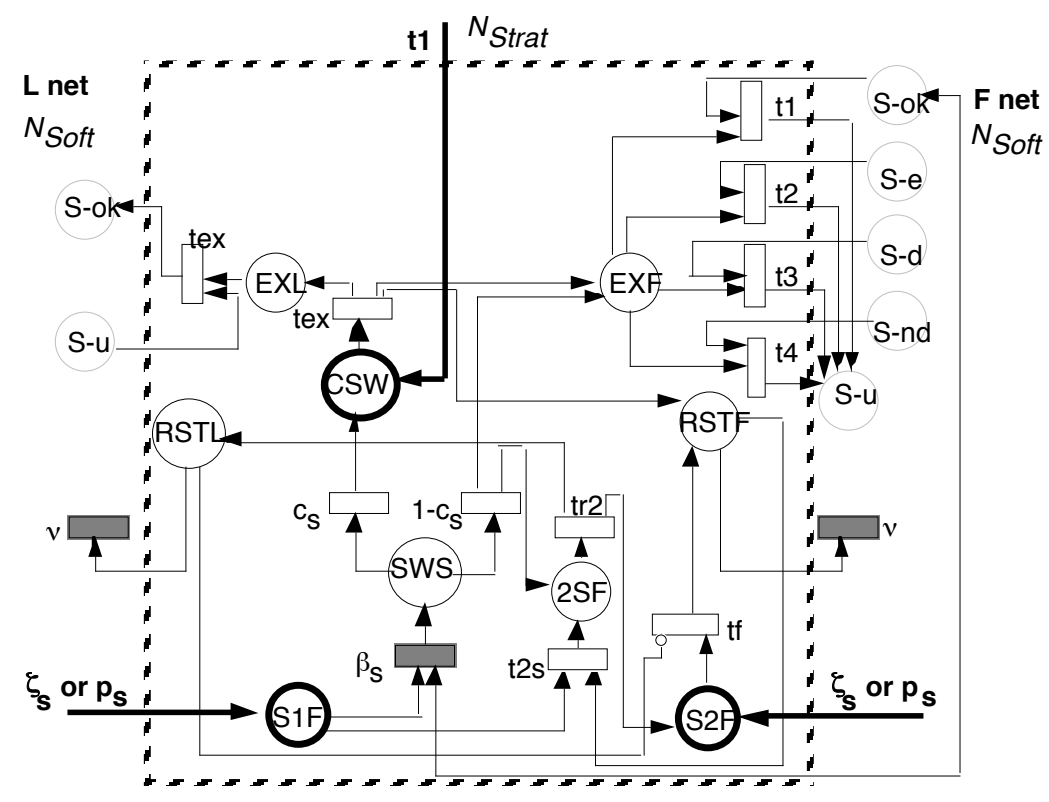

Figure 7: Software reconfiguration net, $N_{R e c}$

- if $\mathrm{F}$ is in failure (RSTF marked) $\mathrm{t} 2 \mathrm{~s}$ is fired and $2 \mathrm{SF}$ is marked allowing the firing of tr2 that marks RSTL and S2F. Marking of place RSTL enables transition $v$ in the leader to restart it. Marking of S2F allows the restart of F only after the end of the leader restart.

- if $\mathrm{S} 2 \mathrm{~F}$ is marked, $\mathrm{F}$ is in failure ( $\mathrm{F}$ is the initializing and the target net ): if $\mathrm{L}$ is not in failure (RSTL not marked) transition tf can be fired and RSTF is marked, authorizing the restart of F; else the token stays in S2F until the end of L restart, restart of F is then allowed via RSTF marking. 
If $N_{R e c}$ is initialed by L only, its is an action and an authorization net. If $N_{\operatorname{Rec}}$ is initialized by $\mathrm{F}$ only, it is an authorization net. If $N_{R e c}$ is initialed by L then F (or F then L) it is an authorization net.

\subsection{Global recovery strategy net}

The global recovery strategy net is initialized by $N_{\text {Rep }}$ through F1H following the firing of tex. If $\mathrm{F}$ is in failure (RSTF marked) $\mathrm{t} 2$ removes the token from RSTF and deposits a token in RSTL and another one in S2F in order for L to be repaired first. If F is not in failure (RSTF not marked) transition t1 deposits a token in CSW in order that the roles of the follower and leader to be exchanged.

$N_{\text {Strat }}$ is an action net if place RSTF is marked and an activation net if RSTF is not marked.

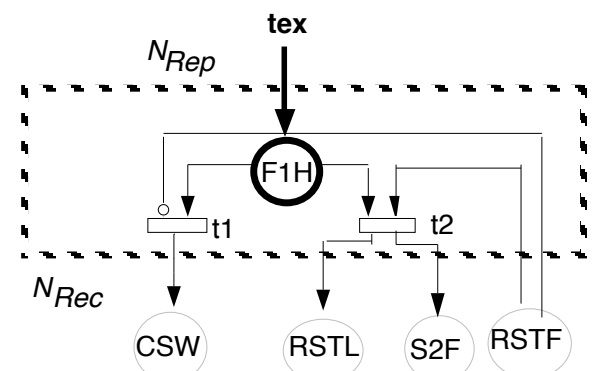

Figure 8: Global recovery strategy net, $N_{\text {Strat }}$

\subsection{Concluding remarks and global model}

It can be checked that the hardware and the software GSPNs are live and bounded. With respect to dependency nets, verification of these properties have to be done progressively. In a first step, each dependency net is validated with the adjacent nets as indicated in figure 2, as follows:

- $\mathrm{N}_{\text {Prop }}$ has to be validated connected with $\mathrm{N}_{\text {Hard }}$ and $\mathrm{N}_{\text {Soft }}$, ( $\mathrm{N}_{\text {Prop }}$ is identical to $\mathrm{N}_{\text {Prop }}$ ),

- $\mathrm{N}_{\text {Stop }}$ has to be validated with $\mathrm{N}_{\text {Hard }}$ and $\mathrm{N}_{\text {Soft }}$,

- $\mathrm{N}_{\text {Rep }}$ has to be validated with two $\mathrm{N}_{\text {Hard }}$,

- $\mathrm{N}_{\mathrm{Rec}}$ has to be validated with $\mathrm{N}_{\text {Soft }}$.

In a second step, all these nets are integrated jointly and validated. The last step consists in adding $\mathrm{N}_{\text {Strat }}$ that has to be validated with all the other nets (which have already been validated together). 
The overall model obtained by replacing the blocks of figure 2 with their GSPNs given in figures 3 to 8 has been processed by SURF-2. The reachability graph has 1200 markings and the Markov chain 500 states without any state aggregation due to symmetry.

It could be argued that the state space may be very large for more complex systems, this is inherent to the complexity of the system to be modeled and to the level of detail considered. The only difficulty due specifically to our modeling approach is the number of markings; it can be overcome by using an aggregation technique at the GSPN level to suppress immediate (see e.g. [1]).

Considering again the duplex system, taking into account the fact that the transition rates associated with error detection and processing mechanisms are very high compared to failure, repair and restart rates (the duration of error detection and processing is of the order of the second whereas the intervals to failures are several hundreds of hours), the model can be reduced to 9 states as shown in figure 9 . This model is to be considered as a limiting case allowing verification of the complete model in this specific case. It allows for instance to check that the stable states of the systems and the transitions between these states actually correspond to what we wanted to model with the GSPN resulting from the composition of the individual component and dependency nets. In the following, some examples of verifications are given:

- States $(2,0,1),(2,1,0)$ and $(2,0,0)$ are failure states due a software failure. It is worth noting that these failures may be due to the propagation of a temporary hardware error (term " $\mathrm{p}_{\mathrm{s}} \mathrm{pr}_{\mathrm{h}}\left(1-\mathrm{p}_{\mathrm{h}}\right)$ $\lambda_{h}$ " in the expressions of $\lambda_{s L}$ and $\lambda_{S F}$ ). Moreover, the latter (state $(2,0,0)$ ) may result from a double propagation of a hardware error to the leader and then to the follower (term " $\mathrm{p}_{\mathrm{s}} \mathrm{pr}_{\mathrm{s}} \mathrm{p}_{\mathrm{s}} \mathrm{pr}_{\mathrm{h}}$ $\left(1-p_{h}\right) \lambda_{h} "$ in the expression of $\left.\lambda_{s L F}\right)$.

- In case of successive failures of the hardware computers, the computers are introduced in sequence whatever is the repair policy adopted, R1 or R2: from states $(0,0,0)$ to $(1,1,0)$ then to $(2,1,1)$. The difference in the two repair policies is that in $\mathrm{R} 2$ the repair rate is $2 \mu$ whereas for $\mathrm{R} 1$ it is $\mu$. 


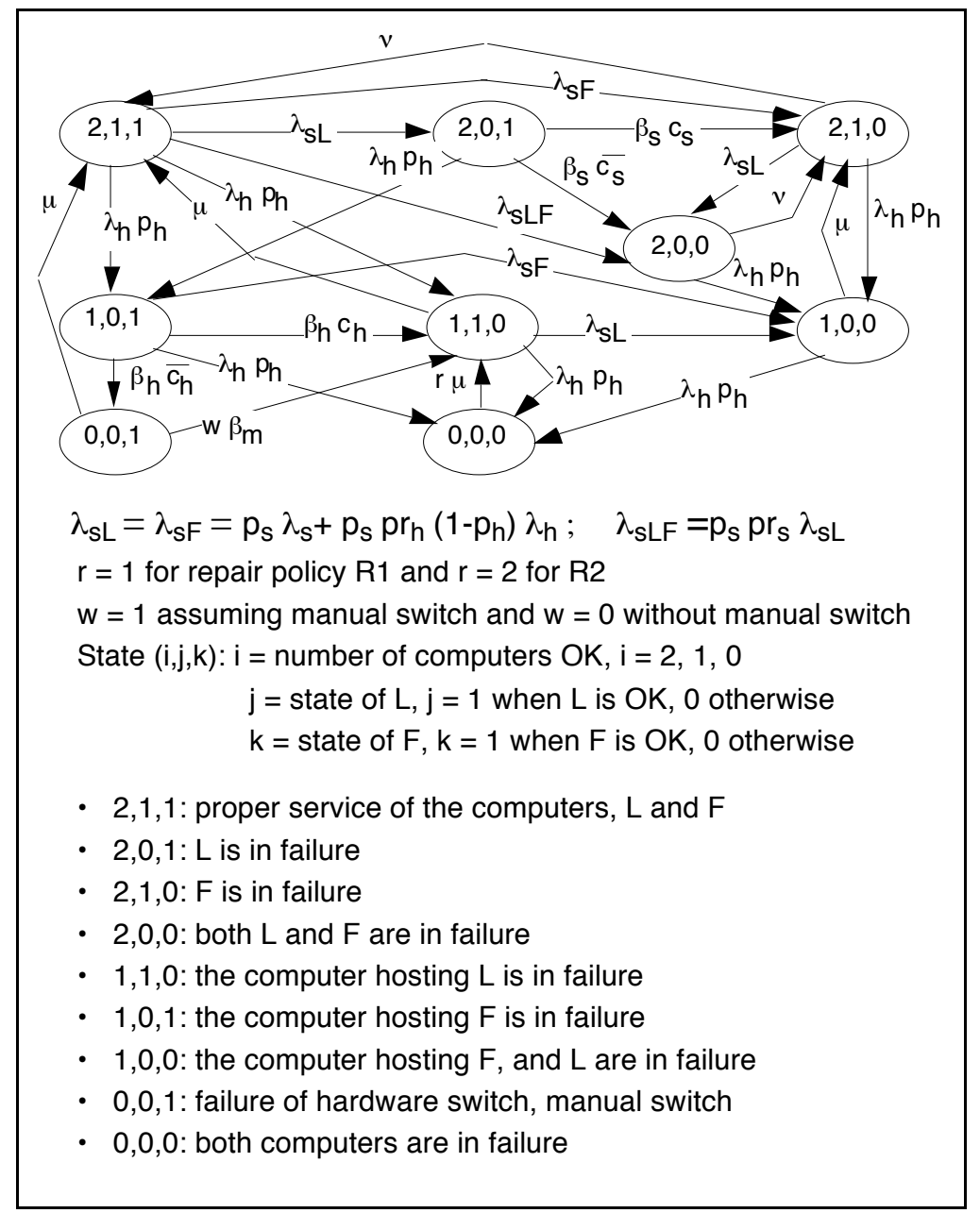

Figure 9: Reduced Markov chain of the duplex system

\section{Conclusion}

The work presented in this paper has allowed various types of dependencies between hardware and software components of a fault-tolerant system to be identified. These dependencies may result from functional or structural interactions as well as interactions due to reconfiguration and maintenance strategies. The dependability model of the system is obtained by composition of the components models with those associated with the dependencies. The rules for interfacing the models have been clearly defined and formally described to build up and easily validable system models. The formal description facilitates the composition of the various GSPNs. 
The modeling approach has been illustrated by a simple example, including all the types of dependencies identified: the duplex system. Modeling of this system showed the strong dependency between components. For example: the activation of a temporary hardware fault, may propagate an error to the hosted software component, which in turn may propagate to other components communicating with it (without being necessarily on the same computer). Thus the activation of a hardware fault, may lead to the restart of one or more software components. Even if this has already been observed on real-life systems, it has not been modeled explicitly in previous work. Also, we have shown how the modification of one or several assumptions can be performed without modifying all GSPNs, considering two repair policies and two switching policies (with or without manual switch).

The main advantage of the modeling approach, based on considering explicitly the interactions, lies in its efficiency for modeling several alternatives for the same system. These alternatives may differ by their composition (number of computers or replicas) or the organization (distribution of software components onto the hardware) or by the fault tolerance and maintenance strategies. One can clearly identify from the beginning the components and interactions that are specific and those that are common to all alternatives. The GSPNs that are common are thus developed and validated only once.

This approach has been applied to the French Air Traffic Control system (the subset associated with the Flight Plan Processing and Radar Data Processing) in [9] where in a first step twelve alternative architectures have been modeled and their unavailability compared to identify the most suitable one. Based on the results of this first step, additional and more detailed architectures have been modeled in [4] to select the architecture with the lowest unavailability. This application showed all the power of the modeling approach with the explicit modeling of the interactions. 


\section{References}

[1] H. H. Ammar, Y. F. Huang and R. W. Liu, "Hierarchical Models for Systems Reliability, Maintainability, and Availability", IEEE Trans. on Circuits and Syst., CAS-34 (6), pp. 629-38, 1987.

[2] G. Balbo, "On the Success of Stochastic Petri Nets", in 6th International Workshop on Petri Nets and Performance Models, (Durham, NC, USA), pp. 2-9, 1995.

[3] C. Béounes, M. Aguéra, J. Arlat, S. Bachman, C. Bourdeau, J. E. Doucet, K. Kanoun, J.-C. Laprie, S. Metge, J. Moreira de Souza, D. Powell and P. Spiesser, "SURF-2: A Program for Dependability Evaluation of Complex Hardware and Software systems", in 23rd IEEE Int. Symp. Fault-Tolerant Computing, (Toulouse, France), pp. 668-73, 1993.

[4] M. Borrel, Interactions between Hardware and Software Components: Characterization, Formalization and Modeling - Application to CAUTRA Dependability, PhD Dissertation, N96-001, In French, 1996.

[5] A. Costes, C. Landrault and J.-C. Laprie, "Reliability and Availability Models for Maintained Systems Featuring Hardware Failures and Design Faults", IEEE Trans. on Computers, C-27 (6), pp. 548-60, 1978.

[6] J. B. Dugan and M. Lyu, "System-level Reliability and Sensitivity Analysis for Three Fault-tolerant Architectures", in 4th IFIP Int. Conference on Dependable Computing for Critical Applications, (San Diego), pp. 295-307, 1994.

[7] W. R. Elmendorf, "Fault-tolerant Programming", in 2nd IEEE Int Symp. Fault-Tolerant Computing, (Newton, Massashusetts), pp. 79-83, 1972.

[8] J. Gray, "Why Do Computers Stop and What Can be Done About it ?", in 5th Int. Symp. on Reliability in Distributed Software and Database Systems, (Los Angeles, CA), pp. 3-12, 1986.

[9] K. Kanoun, M. Borrel, T. Moreteveille and A. Peytavin, "Modeling the Dependability of CAUTRA, a Subset of the French Air Traffic Control System", in 26th Int. Symp. Fault-Tolerant Computing (FTCS26), (Sendai, Japan), pp. 106-15, 1996.

[10] J.-C. Laprie, "Trustable Evaluation of Computer Systems Dependability", in Applied Mathematics and Performance/Reliability Models of Computer/Communication Systems, (Pise, Italie), pp. 341-60, 1983.

[11] J.-C. Laprie (Ed.), Dependability: Basic Concepts and Terminology, Dependable Computing and FaultTolerant Systems, 5, 265 p. , Springer Verlag, Wien-New York, 1992.

[12] J.-C. Laprie, "On The Temporary Character of Operation-persistent Software Faults", in 4th Int. Symp. on Software Reliability Engineering, (Denver, Colorado), pp. 125, 1993.

[13] J.-C. Laprie and K. Kanoun, "X-ware Reliability and Availability Modeling", IEEE Trans. on Software Engineering, SE-18 (2), pp. 130-47, 1992. 
[14] J.-C. Laprie, K. Kanoun, C. Béounes and M. Kaâniche, "The KAT (Knowledge-Action-Transformation) Approach to the Modeling and Evaluation of Reliability and Availability Growth", IEEE Trans. Software Engineering, SE-17 (4), pp. 370-82, 1991.

[15] J. K. Muppala, A. Sathaye, R. Howe, C and K. S. Trivedi, "Dependability Modeling of a Heterogeneous VAX-cluster System Using Stochastic Reward Nets", in Hardware and Software Fault Tolerance in Parallel Computing Systems (D. R. Avresky, Ed.), pp. 33-59, 1992.

[16] P. I. Pignal, "An Analysis of Hardware and Software Availability Exemplified on the IBM-3725 Communication Controller", IBM Journal of Research and Development, 32 (2), pp. 268-78, 1988.

[17] D. Powell, “Distributed Fault Tolerance: Lessons from Delta-4”, IEEE Micro, 14 (1), pp. 36-47, 1994.

[18] W. Sanders and J. Meyer, "Reduced Base Model Construction Methods for Stochastic Activity Networks", IEEE Trans. on Selected Areas in Communications, 9 (1), pp. 25-36, 1991.

[19] D. P. Siewiorek and R. S. Swarz, The Theory and Practice of Reliable System Design, Digital Press, 1992.

[20] G. E. Stark, "Dependability Evaluation of Integrated Hardware/Software Systems", IEEE Trans. on Reliability, R-36 (4), pp. 440-4, 1987. 